\title{
On the Influence of Water-Side Turbulence on an Evaporating Free Surface
}

\author{
William Hay, Miltiadis Papalexandris \\ Universite catholique de Louvain-la-Neuve \\ Institute of Mechanics, Materials and Civil Engineering, Universite catholique de Louvain-la-Neuve \\ Place du levant 2, Louvain-la-Neuve, Belgium \\ william.hay@uclouvain.be; miltos@uclouvain.be
}

\section{Extended Abstract}

The evaporation process from an air-water interface involves a complex collection of inter-dependent phenomena controlled predominantly by the vapour pressure, and hence temperature of the interface, but influenced also by gas and water side convection as well as the means of heat addition. There is currently a lack of data in the literature, both numerical and experimental, for free surface evaporation from a water filled cavity with heat addition from below. For this set-up, and to this authors knowledge, there is no study analysing the effect of water-side turbulence (Rayleigh number) on evaporation rate. A better understanding of the aforementioned conditions are key to an accurate representation of the physical phenomena of a Spent Fuel Pool Loss of Coolant Accident (SFP-LOCA); a fact which motivates this research.

In our presentation the available Sherwood number relations from the literature for evaporative mass transfer are first discussed. References [1] - [3] represent the unique examples where air above the interface is under natural convection and the water temperatures are greater than that of air. The differences in physical phenomena represented by these three references are then discussed, highlighting the need for further research.

Relevant numerical simulation data is also rare due to the fact that combined heat and mass transfer across the interface between a condensed liquid phase into a gaseous mixture of its vapour phase plus air (i.e. multi-component mass transfer) is an ongoing area of research. More specifically, Sherwood number relations are particularly limited for simulations where local volume change is captured, as is required for high mass transfer rates [4]; again a condition associated with SFPLOCAs.

In the first part of the talk we present the governing equations to be solved for the case of turbulent natural convection in a water-filled cavity with mass transfer at the free surface. These include mass, momentum and energy conservation and a single air-side species equation for a bi-component gaseous mixture. The equations required for interface reconstruction and advection are then explained, and jump conditions across said interface are elaborated for the aforementioned governing equations. The numerical algorithm based on the Volume-of-Fluid (VOF) method is then developed for a low-Mach number, non-isothermal flow with interfacial mass transfer in the style of [4-6] but with a novel proposition for a dynamic calculation of evaporation rate.

In the second part of the talk we present numerical results for turbulent natural convection in a differentially heated cavity with a free surface but without evaporation. Large-Eddy Simulations (LES) and Direct Numerical Simulations (DNS) over a range of different Rayleigh numbers are presented and discussed. Emphasis is placed on the analysis of the emerging turbulent structures and, where possible, simulations are compared against available experimental results such as [7]. Our presentation concludes with a discussion on the effect of water side turbulence on free-surface area. Given that evaporation rate is proportional to surface area, it can be insighted that an increase in near-surface turbulence will also increase mass transfer proportionally. The results provide, for the first time, an insight into understanding the role of water-side turbulence on evaporation rates for a water-filled cavity heated from below.

\section{References}

[1] S. M. Bower and J. R.Saylor, "A study of the sherwood-rayleigh relation for water undergoing natural convectiondriven evaporation," International Journal of Heat and Mass Transfer, vol. 52, pp. 3055-3063, 2009. 
[2] L. M. K Boelter, H. S. Gordon and J. R. Griffin, "Free evaporation into air of water from a free horizontal quiet surface," Industrial and Engineering Chemistry, vol. 38, pp. 6, 1946.

[3] L. M. K Boelter and B. F. Sharpley, "Evaporation of water into quiet air from a one-foot diameter surface," Industrial and Engineering Chemistry, vol. 30, p. 10, 1938.

[4] J. Schlottke and B. Weigand, "Direct Numerical Simulation of evaporating droplets," Journal of Computational Physics, vol. 227, pp. 5215-5237, 2008.

[5] S. Fleckenstein and D. Bothe, "A Volume-of-Fluid based numerical method for multi-component mass transfer with local volume changes," Journal of Computational Physics, vol. 301, pp. 35-38, 2015.

[6] M. Irfan and M. Muradoglu, "A front tracking method for Direct Numerical Simulation of evaporation process in a Multiphase System," Journal of Computational Physics, vol. 337, pp. 132-153.

[7] K. A Flack, J. R. Saylor and G. B. Smith, "Near-surface turbulence for evaporative convection at an air/water interface," Physics of Fluids, vol. 13, p. 11, 2001. 